\title{
NOVEDADES TRIBUTARIAS PARA 2014. PRIMEROS FALLOS DE LA SUPREMA CORTE DE JUSTICIA DE LA NACIÓN ${ }^{1}$ Emilio Margáin Manautou ${ }^{2}$
}

\section{Resumen}

En el presente documento se hace referencia a las Novedades Tributarias que entraron en vigor a partir del primero de enero del presente año. Y las primeras resoluciones de la SCJN.

\begin{abstract}
This paper presents and analyzes the new taxes that came into force in the present year. And the first decisions of the Supreme Court .

Sumario. Introducción. II. Facturación Electrónica que Introduce el Legislador en el Código Fiscal de la Federación. III. Contribuciones. Siendo los Derechos. ¿Pueden Incrementarse por un Organismo Descentralizado o por un Secretario de Estado?. IV. Casa Habitación. Su Enajenación No Genera el Impuesto al Valor Agregado. V. Formalidades. Violación a Ellas al Momento de Desahogarse una Orden de Visita Domiciliaría. VI. Orden de Visita Domiciliaria. ¿Puede el Visitador Asentar a Quien Dirigirla?.

VII. Requerimiento de Créditos Fiscales Inoperantes al Haber Transcurrido el Plazo que Señala el Artículo 50 del Código Fiscal de la Federación.
\end{abstract}

\section{Introducción}

De las novedades de las reformas fiscales para el 2014 cabe destacar las siguientes:

En primer término, que se declara constitucional la eliminación del sistema tributario del impuesto sobre la renta de las personas físicas. Régimen de Pequeños Contribuyentes (Repecos), vigente hasta el 31 de diciembre de 2013,

\footnotetext{
${ }^{1}$ Por la importancia del tema se obtuvo la autorización--- para su reproducción en este medio-- -de su autor licenciado Emilio Margáin Manautou del artículo Publicado originalmente en el "Boletín Jurídico" Año 37 Número 438 Despacho Emilio Margáin y Asociados Agosto 2014

2 Distinguido Ex Catedrático de Derecho Fiscal y Administrativo de la Universidad Nacional Autónoma de México y de otras Universidades Públicas y Privadas del País y del extranjero. Reconocido Profesional de los Foros Jurídicos Nacionales y Locales en el ámbito Administrativo y Fiscal. Autor de mas de 20 obras actualizadas cada año en la materia, entre otras, "De lo Contencioso Administrativo", "Introducción al Estudio del Derecho Tributario", "Introducción al estudio del Derecho Administrativo Miembro Invitado Academia de Derecho Administrativo a partir de la entrada en vigor(1991) de la Ley 4 que regula orgánicamente la vida académica de la Universidad de Sonora "
} 
por uno nuevo que será aplicado por el físico federal y que da fin a la corrupción e inmoralidad con que era manejado por los Estados. Lamentablemente es sustituido por uno nuevo que pretende que los contribuyentes menores ---con ingresos anuales hasta dos millones de pesos --- en el que se debe emitir un comprobante fiscal mediante computadora por contribuyentes establecidos en locales fijos o semifijos, expresando, la casi totalidad de ellos, que a duras penas pueden leer y escribir, y quiere el fisco que manejen una computadora. Se alegó por los Repecos, derechos adquiridos para continuar con el sistema derogado, que no se aceptó.

Y el Servicio de Administración Tributaria ha declarado que de no atacarse el nuevo mecanismo de cumplimiento de obligaciones fiscales pasarán a ser considerados obligados a tributar como si fueran contribuyentes ordinarios. Lamentablemente entre los llamados pequeños contribuyentes están disfrazados buenos números de mayores.

En segundo término, el aumento de las tasas a pagar en el impuesto sobre la renta, la Corte confirmó su constitucionalidad, que era de esperarse.

Está en trámite la objeción a la disminución de la prestaciones de previsión social cuya deducibilidad se disminuye para las empresas o contribuyentes que las otorgan.

Tan pronto se publiquen en la Gaceta del Seminario Judicial de la Federación se dará a conocer los fallos correspondientes, con sus comentarios.

\section{Facturación Electrónica que Introduce el Legislador en el Código Fiscal de la Federación.}

A partir del presente año el legislador introduce en el Código Fiscal de la Federación, novedades tributarias que alarmó sensiblemente a los contribuyentes pequeños, medianos e importantes, al no estar preparados para ellas, al alterar el 
mecanismo para emitir la factura o comprobante fiscal por cada operación que realicen por concepto de enajenación de prestación de servicios, uso y/o goce temporal de bienes.

Los contribuyentes menores o pequeños cuyos ingresos anuales no excedan de dos millones de pesos, no pagaran el impuesto sobre la renta durante el presente ejercicio fiscal y por los siguientes ejercicios irán declarando un diez por ciento de ellos hasta que se cumplan diez años. Constituyen el grueso de los contribuyentes, esencialmente personas físicas la mayoría de ellos, siendo la totalidad sujetos hasta el año pasado al sistema de cuota fija que se implantó por primera vez por el fisco federal en el Estado de Sonora en 1961, pero que fue objeto de corrupción por las entidades locales a quienes se les trasladó la atención de estos contribuyentes, que ahora están sujetos al Régimen de Incorporación Fiscal; régimen que por su mecanismo no se cree que se cumpla con él pero que de no acogerlo por los pequeños contribuyentes se les considerará como contribuyentes que deben cumplir como si fueran medianos o importantes.

Para el Régimen de Incorporación Fiscal se pretende que por cada operación que realicen expidan la factura electrónica y como buen número de ellos han expresado: si no leo, ni escribo bien el español cómo piensan que voy a poder manejar una computadora para extraer la nueva factura o bien, el locatario de un mercado público con dos o tres clientes esperando ser atendidos, se les tendrá que pedir que tengan la paciencia de esperar en tanto surja la factura que deberá elaborar.

Piénsese también en los contribuyentes de puestos semifijos. ¿Podrá la mayoría adquirir una computadora y aprender a emitir la factura electrónica?

Serios problemas tendrán las autoridades fiscales con éstos contribuyentes. 
Pero bien puede sostenerse que un diez porciento de los locatarios de mercados públicos y de los llamados "mercados sobre ruedas" son importantes disfrazados de pequeños contribuyentes.

Con la creación de la factura electrónica se ha complicado extraordinariamente el cumplimiento de las obligaciones fiscales y llama la atención que empresas trasnacionales, en servicios secundarios que prestan aclaran que el pago debe ser en efectivo y no con cheque nominativo.

Hasta han surgido Proveedores Autorizados de Certificación (PAC) por el Servicio de Administración Tributaria (SAT)

Se ha expresado que "las decisiones que el SAT toma en materia de facturación electrónica no solamente cambia el panorama para los contribuyentes, también para los PAC, que están a cargo de proveer los sistemas, el timbrado y almacenamiento para las facturas electrónicas, deben prepararse para el cambio ${ }^{3}$

EI SAT ha venido difiriendo la vigencia de la emisión de facturas electrónicas, aun cuando hay un buen número de contribuyentes que sí están cumpliendo. Sin embargo, se han promovido miles de amparos en contra de las disposiciones del Código Fiscal de la Federación y de la Key del Impuesto sobre la Renta para combatir las novedades fiscales que afectan a los contribuyentes no únicamente incumplidos sino también a los cumplidos.

Por qué el fisco mexicano no hizo primero lo que otros fiscos, hoy avanzados, hicieron en el pasado: combatir la evasión tributara y una vez que habían reducido ese problema en un $85 \%$, entonces modificaron su sistema tributario. Tal parece que el fisco federal pretende tratar de hacer lo segundo sin atacar previamente la

\footnotetext{
${ }^{3}$ Soto Gonzalo, "Acusan a Corte de favorecer al fisco"periódico REFORMA, Sección Negocios 13 de junio de 2013[consultado el 31 de julio de 2014http://www.negociosreforma.com/aplicacioneslibre/preacceso/articulo/default.aspx?]
} 
evasión tributaria y reducir la corrupción que se padece que influye en aquella, tanto por personal administrativo como por terceros ajenos a la administración.

\section{III.Contribuciones. Siendo los Derechos. ¿Pueden Incrementarse por un Organismo Descentralizado o por un Secretario de Estado?}

El artículo 2, fracción IV, del Código Fiscal de la Federación, establece:

"Artículo $2^{\circ}$.- Las contribuciones se clasifican en impuestos, aportaciones de seguridad social, contribuciones de mejoras y derechos, las que se definen de la siguiente manera:

IV. Derechos son las contribuciones establecidas en Ley por el uso o aprovechamiento de los bienes del dominio público de la Nación, así como por recibir servicios que presta el Estado en sus funciones de derecho público, excepto cuando se presten por organismos descentralizados $u$ órganos desconcentrados cuando en este último caso, se trate de contraprestaciones que no se encuentren previstas en la Ley Federal de Derechos. También son derechos las contribuciones a cargo de los organismos públicos descentralizados por prestar servicios exclusivos del Estado"

Con motivo de la construcción del nuevo aeropuerto internacional de la Ciudad de México NAICM que se iniciará próximamente en el mes de enero del 2015, se ha publicado en los periódicos que los pasajeros pagarán un 40\% más por concepto de Tarifa Única por uso de Aeropuerto (TUA), con lo cual los usuarios del Aeropuerto Internacional de la Ciudad de México cooperarán con el $40 \%$ del costo del aeropuerto que se construirá en terrenos del Lago de Texcoco en el Estado de México.

Siendo una contribución los derechos que nuestro Código Fiscal de la Federación asigna por el uso o aprovechamiento de los bines del dominio público de la Nación, más conocida por la doctrina y legislaciones extranjeras como tasa, se requiere que el incremento del TUA sea aprobada por el Congreso de la Unión y promulgada dicha aprobación por el Presidente de la República, que al ser aprobada por el Congreso de la Unión se incremente algo de lo que se tenga 
pensado o bien hasta que esté operando el Nuevo Aeropuerto Internacional de la Ciudad de México NAICM que será en unos cuatro o más años.

\section{IV.Casa Habitación. Su Enajenación No Genera el Impuesto al Valor Agregado.}

El artículo 9, fracción II, de la Ley del Impuesto al Valor Agregado, establece lo siguiente:

Artículo 9o.-No se pagará el impuesto en la enajenación de los siguientes bienes:

II.-Construcciones adheridas al suelo, destinadas o utilizadas para casa habitación. Cuando sólo parte de las construcciónes se utilicen o destinen a casa habitación, no se pagará el impuesto por dicha parte. Los hoteles no quedan comprendidos en esta fracción.

De lo transcrito se observa que la enajenación de casa habitación para estar exenta no está sujeta a condición o a requisito alguno de utilizarse el inmueble exclusivamente como casa habitación.

Sin embargo, el artículo 21-A del reglamento de dicha Ley, vigente hasta el 4 de diciembre de 2006, expresaba que la enajenación de una casa habitación no quedaba gravada si quien la enajenaba había proporcionado la mano de obra e insumos, esto es, establecía una condición no prevista en la Ley de la materia. Por lo tanto, si quien poseía una casa o unidad habitacional la construyó mediante contrato de obra a precio alzado, se tenía que pagar el impuesto al enajenarse al año o más por cuanto que e enajenante no había proporcionado la mano de obra e insumos, sino quien construyó la obra.

Solo que la obra se hubiese construido mediante contrato de prestación de servicios, su enajenación estaba exceptuado de pagar el IVA. Criterio erróneo, por cuanto que la Ley no preveía tal condición y un ordenamiento de menor jerarquía, 
como lo es un reglamento de ejecución, no puede modificar la Ley o imponer modalidades que la Ley no contempla.

Sin embargo, tal absurdo jurídico se corrigió al derogarse el artículo 21-A del Reglamento de la Ley del Impuesto al Valor Agregado y, sin embargo, tres señores Ministros de la Segunda Sala establecieron, por mayoría de votos, la siguiente jurisprudencia.

VALOR AGREGADO. LA EXENCIÓN PREVISTA EN LOS ARTÍCULOS 9o. DE LA LEY DEL IMPUESTO RELATIVO Y 21-A DE SU REGLAMENTO (VIGENTE HASTA

EL 4 DE DICIEMBRE DE 2006), OPERA RESPECTO DE QUIENES ENAJENEN CONSTRUCCIONES ADHERIDAS AL SUELO DESTINADAS A CASA HABITACIÓN, CUANDO PROVEEN EN ELLA MANO DE OBRA E INSUMOS.

De la exposición de motivos de la Ley del Impuesto al Valor Agregado, así como del proceso legislativo correspondiente, se concluye que el propósito del creador de la norma fue exentar del pago de ese impuesto únicamente la enajenación de construcciones adheridas al suelo destinadas a casa habitación, quedando incluidas en ese concepto las instalaciones adheridas a la construcción en forma permanente, de manera que no puedan separarse sin causar daño material o estético a la construcción y cuya finalidad sea hacerla funcional para destinarla a una vivienda digna y decorosa, como pueden ser las instalaciones hidráulicas, eléctricas, gas, aire acondicionado y otras; sin embargo, para que opere tal exención es necesario que esa actividad se desarrolle integralmente, es decir, que los actos de enajenación de bienes destinados a casa habitación se realicen en forma conexa con la prestación de servicios de construcción de dichos inmuebles, como pueden ser la mano de obra e insumos necesarios, excluyendo por tanto los actos de adquisición de bienes o servicios, productos semiterminados o terminados y demás insumos estrictamente indispensables para la realización de la obra. Contradicción de tesis 205/2010._Entre las sustentadas por los Tribunales Colegiados Primero, Tercero, Cuarto, Sexto y Décimo Quinto, todos en Materia Administrativa del Primer Circuito.-14 de julio de 2010.-Mayoría de tres votos.Disidentes: Margarita Beatriz Luna Ramos y Luis María Aguilar Morales-.

La derogación de dicho precepto vino a quitarles un dolor de cabeza a las empresas constructoras de unidades habitacionales que se enajenan en condominio. 
Esta increíble jurisprudencia de la Segunda Sala de La Suprema Corte de Justicia de la Nación, 115/2010, visible en el seminario Judicial de la Federación y su Gaceta, Novena Época, Tomo XXXII, Agosto de 2010, página 445, se apoyó en lo que expresaba el artículo 21-A del Reglamento de la Ley del Impuesto al Valor Agregado, vigente en 2006, párrafo primero, que sostenía.

"Artículo 21-A- La prestación de los servicios de construcción de inmuebles destinados a casa habitación, ampliación de ésta, así como la instalación de casas prefabricadas que sean utilizadas para este fin, se consideran comprendidos dentro de lo dispuesto por la fracción II del artículo $1^{\circ}$.de la Ley, siempre y cuando el prestador del servicio proporcione la mano de obra y materiales".

Esto es, sí la casa habitación la construye el propio interesado, poniendo él la mano de obra y los insumos, al enajenarla tendría que trasladar al adquiriente el impuesto al valor agregado, es decir, se hace una distinción que la ley no hace y en un ordenamiento de interior jerarquía jurídica a la Ley de la materia.

\section{Formalidades. Violación a Ellas al Momento de Desahogarse una Orden de Visita Domiciliaría.}

El Primer Tribunal Colegiado en Materia Administrativa del Tercer Circuito, emitió la tesis aislada III. $1^{\circ}$.A.165 A, Visible en el Semanario Judicial de la Federación y su Gaceta, Novena Época Tomo XXXIII, Mayo de 2011, página 1238, que nos dice:

ORDEN DE VISITA DOMICILIARIA. LA PROHIBICIÓN DE EMITIR UNA NUEVA, RELATIVA A LOS MISMOS HECHOS REVISADOS, ESTABLECIDA EN EL ARTÕCULO 46, ÚLTIMO PÁRRAFO, DEL CÓDIGO FISCAL DE LA FEDERACIÓN, NO SE ACTUALIZA CUANDO EL MANDAMIENTO SE DICTA PARA CORREGIR UNA RESOLUCIÓN 


\section{ORIGINADA POR AQUÉLLOS, DECLARADA NULA POR VICIOS DE}

FORMA.- El ultimo párrafo del articulo 46 del Código Fiscal de la Federación, reformado mediante decreto publicado en el Diario Oficial de la Federación el 10. de octubre de 2007, en vigor a partir del 10. de enero del año siguiente, establece que concluida la visita domiciliaria podrá efectuarse una nueva a la misma persona, respecto de las mismas contribuciones, aprovechamientos y periodos, posibilidad que el propio numeral limita a la comprobación de hechos diferentes a los ya revisados. Así, de una interpretación a contrario sensu, se advierte que dicho precepto prohíbe que se emita una nueva orden relativa a los mismos hechos, es decir, a los que están sometidos a un juicio en trámite, que hayan sido objeto de nulidad en términos absolutos o definitivos, o que fueron sustento de una determinación que quedó firme, lo cual no se actualiza cuando aquéllos dieron origen a una resolución declarada nula por vicios de forma, porque, en este caso, la autoridad conserva sus facultades para corregirla basándose en los mismos hechos, pues éstos no han dado sustento a un pronunciamiento definitivo $y$, por tanto, aquélla puede dictar otra orden para subsanar el error, la cual no debe calificarse como "nueva", prohibida por la norma, sino sólo como "otra" sustitutiva.

El último párrafo del artículo 46 del Código Fiscal de la Federación, vigente en 2011, expresaba lo siguiente:

"Concluida la visita en el domicilio fiscal, para iniciar otra a la misma persona, se requerirá nueva orden. En el caso de que las facultades de comprobación se refieran a las mismas contribuciones, aprovechamientos y períodos, solo se podrá efectuar la nueva revisión cuando se comprueben hechos diferentes a los ya revisados. La comprobación de hechos diferentes deberá estar sustentada en información, datos o documentos de terceros, en la revisión de conceptos específica que no se hayan revisado con anterioridad, en los datos aportados por los particulares en las declaraciones complementarias que se presenten o en la documentación aportada por los contribuyentes en los medios de defensa que promueven y que no hubiera sido exhibida ante las autoridades fiscales, durante el ejercicio de las facultades de comprobación previstas en las disposiciones fiscales; a menos que en este último supuesto la autoridad no haya objetado de falso el documento en el medio de defensa correspondiente pudiendo haberlo hecho o bien, cuando habiéndolo objetado,el incidente respectivo haya sido declarado improcedente".

A partir del $1^{\circ}$ de enero de 2014, la fracción VIII y último párrafo quedan como sigue:

"VIII Cuando de la revisión de las actas de visita y demás documentación vinculada a éstas, se observe que el procedimiento no se ajustó a las 
normas aplicables, que pudieran afectar la legalidad de la determinación del crédito fiscal, la autoridad podrá de oficio, por una sola vez, reponer el procedimiento, a partir de la violación formal cometida.

Lo señalado en la fracción anterior, será sin perjuicio de la responsabilidad en que pueda incurrir el servidor público que motivó la violación...".

Pero si la violación al procedimiento se efectuó al presentarse los visitadores por primera vez a levantar el acta de inicio y han transcurrido más de cinco años al momento de levantarse de nuevo el acta de inicio, ¿está en tiempo la autoridad fiscal para iniciar de nuevo la visita domiciliaria a pesar de que han caducado las facultades de la autoridad fiscal en los términos del artículo 67 del Código Fiscal de la Federación?

\section{Orden de Visita Domiciliaria. ¿Puede el Visitador Asentar a Quien Dirigirla?}

Buen número de órdenes de visita domiciliaria se expedían por autoridad competente sin precisar a quien iba dirigida, lo cual indicaban que se dejaba al criterio del visitador asentar a quien se ordenaba, al implantar de su puño y letra tal dato; visitas éstas que se han eliminado quizás por el estudio que apareció en este BOLETÍN JURÍDICO, que, pretender percibir \$12,000 por emisión incorrecta del comprobante fiscal, generando ello al Estado un gasto de $\$ 70,000.00$ a $\$ 90,000.00$.

El nombre del visitado debía precisarse y así lo consideró el Primer Tribunal Colegiado en Materia Administrativa y del Trabajo del Décimo Sexto Circuito, en la siguiente tesis de jurisprudencia XVI. $1^{\circ}$.A.T. J/22

VISITA DOMICILIARIA. LA ORDEN RELATIVA EMITIDA CON FUNDAMENTO EN LOS ARTÍCULOS 42, FRACCIÓN V Y 49 DEL CÓDIGO FISCAL DE LA FEDERACIÓN, DEBE CONTENER EL NOMBRE DEL CONTRIBUYENTE VISITADO. 
Cuando una visita domiciliaria tenga como finalidad verificar la documentación o los comprobantes que acrediten la legal propiedad, posesión, estancia, tenencia o importación de mercancías y se fundamente en los artículos 42, fracción V y 49 del Código Fiscal de la Federación, a fin de respetar la inviolabilidad del domicilio, como un derecho subjetivo del gobernado, la orden relativa debe contener el nombre del contribuyente visitado, acorde con lo sostenido por la Segunda Sala de la Suprema Corte de Justicia de la Nación en la jurisprudencia 2a./J. 57/99, publicada en el Semanario Judicial de la Federación y su Gaceta, Novena Época, Tomo IX, junio de 1999, página 343, de rubro: "VISITA DOMICILIARIA. LA ORDEN EMITIDA PARA VERIFICAR EL CUMPLIMIENTO DE LAS OBLIGACIONES EN RELACIÓN CON LA EXPEDICIÓN DE COMPROBANTES FISCALES, DEBE PRECISAR EL PERIODO SUJETO A REVISIÓN.", en el sentido de que las órdenes de visita a que se refiere, han de satisfacer el requisito inserto en el artículo 38, fracción $\mathrm{V}$, del señalado código, así como los que establece el artículo 16 de la Constitución Política de los Estados Unidos Mexicanos para los cateos, entre otros, el nombre del sujeto pasivo visitado, sin que sea aplicable el precepto 43, fracción III, en relación con el 44 , ambos del indicado código, que eximen de esa exigencia cuando se trate de órdenes de verificación en materia de comercio exterior, al tratarse de un procedimiento distinto al inicialmente descrito.- Visible en el SJF y G, Novena Época, tomo XXXIII, Marzo de 2011, pág. 2249-.

\section{Requerimiento de Créditos Fiscales Inoperantes al Haber Transcurrido el Plazo que Señala el Artículo 50 del Código Fiscal de la Federación.}

El artículo 50, primer párrafo del Código Fiscal de la Federación, establece lo siguiente:

Artículo 50.Las autoridades fiscales que al practicar visitas a los contribuyentes o al ejercer las facultades de comprobación que se refiere el artículo 48 de este Código, conozcan de hechos u omisiones que entrañen incumplimiento de las disposiciones fiscales, determinarán las contribuciones omitidas mediante resolución que se notificará personalmente al contribuyente o por medio del buzón tributario, dentro de un plazo máximo de seis meses contado a partir de la fecha en que se levante el acta final de la visita $o$, tratándose de la revisión de la contabilidad de los contribuyentes que se efectúe en las oficinas de las autoridades fiscales, a partir de la fecha en que concluyan los plazos a que se refieren las fracciones VI y VII del artículo 48 de este Código. 
Cuando se presentan estos casos la autoridad fiscal procede a requerir el pago del crédito fiscal como si se hubiera notificado la resolución o actuado en tiempo y el contribuyente paga, ignorando las dos jurisprudencias de la Segunda Sala de la SCJN, que los requerimientos son impugnables. Tal proceder de la autoridad es con el objeto de que el contribuyente, sabedor que ha incurrido en omisiones procede en algunos casos a pagar.

El problema que también se presenta es de que, salvo excepciones, el grueso de las Salas Regionales del Tribunal Federal de Justicia Fiscal y Administrativa rechazaban como no impugnables los requerimientos de las autoridades fiscales no obstante las jurisprudencias de nuestro más Alto Tribunal.

Ventilado este problema, la Segunda Sala de la SCJN estableció la siguiente jurisprudencia $2^{a}$./J.211/2007, recaída en la Contradicción de tesis 179/2007-SS, 00 visible en el Seminario Judicial de la Federación, Novena Época, Tomo XXXVI, Diciembre de 2007, página 209.

NOTIFICACIÓN DE LA RESOLUCION QUE DETERMINA UN CRÉDITO FISCAL. LA DECLARATORIA DE SU ILEGILADIDAD Y SU CONOCIMIENTO DESPUÉS DEL PLAZO PREVISTO POR EL ARTÍCULO 50 DEL CÓDIGO FISCAL DE LA FEDERACIÓN (VIGENTE HASTA EL 31 DE DICIEMBRE DE 2003), TIENE EL ALCANCE DE DEJAR SIN EFECTOS LA RESOLUCIÓN Y LAS ACTUACIONES DERIVADAS DE LA VISITA O REVISION.- Conforme a lo previsto por el artículo 50 del Código Fiscal de la Federación, vigente hasta el treinta y uno de diciembre de dos mil tres, en el sentido de que, quedará sin efectos la orden y las actuaciones derivadas de la visita o revisión relativa, corresponde cuando no se emite y notifica en forma personal la resolución administrativa en el plazo de seis meses, lo cual significa que si en virtud de un juicio de nulidad se determina de ilegal la notificación practicada, respecto de la resolución liquidatoria y, por ende el contribuyente conoce el crédito respectivo fuera del término antes aludido, tiene como consecuencia de facto que quede sin efectos la propia resolución administrativa y las actuaciones derivadas de la misma. Lo anterior es así, habida cuenta que los plazos para emitir y notificar en forma personal la resolución liquidadora no fueron cumplidos dentro del término de seis meses; en tanto que el contribuyente conoció la resolución liquidadora fuera del plazo que para ese efecto establece la ley. 


\section{A manera de Conclusiones}

Si bien se declara constitucional la eliminación del sistema tributario del impuesto sobre la renta de las personas físicas ---Régimen de Pequeños Contribuyentes--(Repecos), vigente hasta el 31 de diciembre de 2013, por uno nuevo que será aplicado por el físico federal, lamentablemente se pretende que los contribuyentes menores establecidos en locales fijos o semifijos ---con ingresos anuales hasta dos millones de pesos --- emitan comprobantes fiscales mediante computadora , cuando la casi totalidad de ellos, que a duras penas pueden leer y escribir, quiere el fisco que manejen una computadora..

Y el Servicio de Administración Tributaria ha declarado que de no atacarse el nuevo mecanismo de cumplimiento de obligaciones fiscales pasarán a ser considerados obligados a tributar como si fueran contribuyentes ordinarios. Lamentablemente entre los llamados pequeños contribuyentes están disfrazados buenos números de mayores.

La Suprema Corte confirmó la constitucionalidad del aumento de las tasas a pagar en el Impuesto Sobre la Renta.

Está en trámite la objeción a la disminución de la prestaciones de previsión social cuya deducibilidad se disminuye para las empresas o contribuyentes que las otorgan.

\section{Bibliografía y Webgrafía}

SOTO, Gonzalo, "Acusan a Corte de favorecer al fisco"periódico REFORMA, Sección Negocios 13 de junio de 2013 [consultado el 31 de julio de 2014http://www.negociosreforma.com/aplicacioneslibre/preacceso/articulo/default .aspx?] 
Jurisprudencia $2^{\mathrm{a}}$./J.211/2007, recaída en la Contradicción de tesis 179/2007-SS, 00 visible en el Seminario Judicial de la Federación, Novena Época, Tomo XXXVI, Diciembre de 2007, página 209.

Jurisprudencia XVI. $1^{\circ}$.A.T. J/22. Primer Tribunal Colegiado en Materia Administrativa y del Trabajo del Décimo Sexto Circuito

Jurisprudencia de la Segunda Sala de La Suprema Corte de Justicia de la Nación, 115/2010, visible en el seminario Judicial de la Federación y su Gaceta, Novena Época, Tomo XXXII, Agosto de 2010, página 445.

Tesis aislada III. $1^{\circ}$.A.165 A, Visible en el Semanario Judicial de la Federación y su Gaceta, Novena Época Tomo XXXIII, Mayo de 2011, página 1238, Primer Tribunal Colegiado en Materia Administrativa del Tercer Circuito.

\section{Legislación}

Código Fiscal de la Federación Ley del Impuesto Sobre la Renta Ley del Impuesto al Valor Agregado Reglamento de la Ley del Impuesto al Valor Agregado, 